\section{PILOT STUDY COMPARING SELF-COLLECTED VAGINAL SWAB WITH CLINICIAN TAKEN VAGINAL SWAB FOR THE DETECTION OF CANDIDA AND BACTERIAL VAGINOSIS}

Pam Barnes*, Rute Vieira, Mayur Chauhan. Newcastle upon Tyne Hospitals NHS Foundation Trust, Newcastle upon TYne, UK

\subsection{6/sextrans-2016-052718.19}

Background/introduction Vaginal discharge and vulvitis are common presenting symptoms in both sexual health services and general practice. Due to various constrains particularly in general practice, examination of a patient may not be possible. Syndromic management is often practiced but can be unreliable. Few studies to date have specifically looked at the validity of self-collected vulvovaginal swab for the diagnosis of bacterial vaginosis (BV) and vulvovaginal candidiasis (VVC)

Aim(s)/objectives To describe agreement between self-collected vulvovaginal swabs and clinician taken high vaginal swabs for the detection of BV and VVC.

Design Case controlled study with the patient acting as her own control.

Setting

An urban sexual health centre. Participants: Women aged 1665 years attending with symptomatic vaginal discharge, vulval irritation or an offensive genital smell. Interventions: Participants took a vulvovaginal swab prior to speculum insertion and vaginal examination during which a clinician took a high vaginal swab. Main outcome measure: Diagnosis of BV or VVC infection with samples analysed in a microbiology department using both microscopy and culture.

Results 104 women were enrolled in the study. Of these 45 were diagnosed with VVC. 26 were diagnosed with BV. Using the reference standard of laboratory testing, the sensitivities of self-collected vulvovaginal swabs for BV and VVC were $88.5 \%$ and $95.5 \%$ respectively. The Cohen Kappa score showed strong agreement for the detection of both BV and VVC $(k=0.842$ and $\mathrm{k}=0.878$ respectively).

Discussion/conclusion Self-collected vulvovaginal swabs appear to be a valid alternative to clinician taken high vaginal swabs for detecting BV and VVC infections.

\section{O021 A QUESTION OF STABILITY}

${ }^{1}$ Hemanti Patel ${ }^{*},{ }^{2}$ Gabriel Schembri, ${ }^{3}$ Binta Sultan, ${ }^{4}$ Catherine Ison. ${ }^{1}$ Public Health England, London, UK; ${ }^{2}$ Manchester Centre for Sexual Health, Central Manchester University Hospitals NHS Foundation Trust, Manchester, UK; ${ }^{3}$ Mortimer Market Centre, Central and North West London NHS Foundation Trust, London, UK; ${ }^{4}$ Bacterial Special Interest Group, London, UK

\subsection{6/sextrans-2016-052718.20}

Background/introduction Urines to be tested by the APTIMA Combo 2 (AC2) are added to a collection tube containing preservatives to ensure stability of the nucleic acid for testing within 24 hours of collection. Home collected urines are often collected in containers without preservative to avoid the patient manipulating the sample.

Aim(s)/objectives An investigation was undertaken to determine the stability of gonococcal and chlamydial nucleic acids within neat urine stored in different conditions over a period of 25 days to provide evidence of the stability of the nucleic acid prior to testing.

Methods To mimic collection in a home setting and differing nucleic acid loads within clinical specimens, uninfected urine was inoculated with different concentrations of chlamydial (from cell culture) and gonococcal (from bacterial culture) nucleic acid. Aliquots of the urine were removed on eight occasions over 25 days, added to collection tubes and tested either on the Hologic Panther system to determine presence of RNA or, following DNA extraction, using in-house PCRs to determine DNA load.

Results Chlamydial RNA and DNA remained stable for over three weeks when either refrigerated or stored at room temperature. Gonococcal RNA was detectable up to three weeks if refrigerated and two weeks if stored at room temperature. GC DNA was detectable for 18 days if refrigerated and for 11 days if stored at room temperature.

Discussion/conclusion Chlamydial and gonococcal nucleic acids are stable in urine before addition to preservatives for longer than recommended by the manufacturer, enabling more flexibility for home collected samples.

\section{RECTAL CHLAMYDIA INFECTION IN WOMEN - HAVE WE BEEN MISSING THE POINT?}

${ }^{1}$ Harriet Wallace*, ${ }^{1}$ Michelle Loftus-Keeling, ${ }^{2}$ Helen Ward, ${ }^{3}$ Claire Hulme, ${ }^{4}$ Mark Wilcox, 'Janet Wilson. 'Leeds Sexual Health, Leeds Teaching Hospitals Trust, Leeds, UK; ${ }^{2}$ Department of Infectious Disease Epidemiology, Imperial College, London, UK; ${ }^{3}$ Academic Unit of Health Economics, University of Leeds, Leeds, UK; ${ }^{4}$ Department of Clinical Microbiology, Leeds Teaching Hospitals Trust, Leeds, UK

\subsection{6/sextrans-2016-052718.21}

Background/introduction BASHH standards recommend rectal chlamydia sampling in women with increased risk. However, studies show high rates of rectal chlamydia in women, with concerns over treatment failures and risk of genital re-infection Aim(s)/objectives To determine if rectal chlamydia screening in females should be universal.

Methods As part of a selfswab versus clinician trial we asked females about frequency of vaginal, receptive anal, and oral sex, and correlated this with chlamydia NAATs from these sites.

Results Recruitment to February 2016 included 1041 women. All consented to rectal sampling; none had rectal symptoms. 53\% reported no prior receptive anal sex. 204 women had chlamydia (CT) positive NAATs at one or more sites: 176 (16.9\%) VVS positive (86\% of all CT positives); 190 (18.3\%) rectal positive (93\% of total CT positives); 49 (4.7\%) pharyngeal positive. Rectal swabs were significantly more likely to detect CT than VVS: OR 2.75 (95\% CI 1.22-6.18) p $=0.02$ McNemar test. The table shows percentage women by positive site(s) reporting no anal sex. 92/190 (48.4\%) of those with one site or combination rectal CT reported no previous anal sex. Of the 168 with

\begin{tabular}{|c|c|c|}
\hline $\begin{array}{l}\text { Site(s) of chlamydia } \\
\text { positive NAATs }\end{array}$ & $\begin{array}{l}\text { Number confirmed positive } \\
\text { by site(s) [total 204] }\end{array}$ & $\begin{array}{l}\text { Percentage women with infection } \\
\text { at site(s) reporting never having } \\
\text { had receptive anal sex (\%) }\end{array}$ \\
\hline VVS only & 7 & 43 \\
\hline VVS and rectal & 132 & 50 \\
\hline VVS, rectal, pharyngeal & 36 & 47 \\
\hline Rectal only & 17 & 41 \\
\hline Rectal and pharyngeal & 5 & 40 \\
\hline Pharyngeal and VVS & 1 & 100 \\
\hline Pharyngeal only & 6 & 0 \\
\hline
\end{tabular}


VVS and rectal positive NAATs, the AC2 Reactive Light Units levels were equivalent, suggesting active infection at both sites. Discussion/conclusion In this sample of women with no rectal symptoms, the rectum was the most prevalent site for chlamydia infection, and rectal swabs found significantly more infections than VVS. There was no association with reported anal sex indicating sexual risk history is unreliable for targeted screening in women.

\section{FEASIBILITY STUDY TO DETERMINE THE TIME TAKEN FOR NAATS TESTS TO BECOME NEGATIVE FOLLOWING TREATMENT FOR CHLAMYDIA TRACHOMATIS AND NEISSERIA GONORRHOEAE IN MEN AND WOMEN}

\footnotetext{
1,2Binta Sultan*, 'Clare Oakland, ${ }^{1}$ Nataliya Brima, ${ }^{3}$ Hemanti Patel, ${ }^{1}$ Andrew Copas, ${ }^{2}$ Paul Benn, ${ }^{3}$ Cathy Ison, ${ }^{4}$ Gabriel Schembri. 'University College London, London, UK; ${ }^{2}$ Mortimer Market Centre, London, UK; ${ }^{3}$ Public Health England, London, UK; ${ }^{4}$ Manchester Centre for Sexual Health, Manchester, UK
}

\subsection{6/sextrans-2016-052718.22}

Background/introduction Few data are available to guide the best time to perform a test of cure using nucleic acid amplification tests (NAATs) following treatment for chlamydia (CT) and gonorrhoea (NG).

Aim(s)/objectives The association between the type of infection, organism load, site of infection and treatment were compared to the time for the NAAT to become negative after treatment.

Methods Individuals who had a positive NAAT test for CT and/ or NG were eligible. Self-taken specimens from the site of infection were collected at 8 time points. The time to first negative test following treatment was examined using survival analysis techniques.

Results 102 men (87 MSM) and 52 women were recruited to the study (84 NG, $71 \mathrm{CT}$ infections). 28 participants with NG and 16 with CT were lost to follow up. On day 0, 20 participants diagnosed with NG and 8 diagnosed with CT had negative tests. Median time to negativity for $\mathrm{NG}$ infection was 2 days (IQR 1-5) and for CT infection was 4 days (IQR 2-5). At day 14 after treatment $92 \%$ of participants were CT negative, and $84 \%$ NG negative.All tests were negative by day 35 for both infections.

Discussion/conclusion This study provides valuable data in determining the time to test of cure for CT and NG infections. Site of infection may have an effect on time to clearance of infection, with pharyngeal NG infections and vaginal CT infections taking longer to clear than other sites. The results of this study will help guide clinicians to the timing for test of cure.

\section{TRICHOMONAS VAGINALIS - TREATMENT AND TEST OF CURE ANALYSIS IN A GUM CLINIC POPULATION}

Gabriella Bathgate*, Melissa Perry, John White. Guy's \& St Thomas' NHS Foundation Trust, London, UK

\subsection{6/sextrans-2016-052718.23}

Background/introduction Trichomonas vaginalis is prevalent in patients of black ethnicity in our south London population. Nucleic acid amplification testing (NAAT) is the diagnostic gold standard, and first-line treatment with metronidazole or tinidazole regimens thought to achieve comparable cure rates $>90 \%$. Test of cure (TOC) is recommended if symptoms persist following treatment, but this overlooks persistent asymptomatic infection and optimal timing and testing modality are uncertain. Aim(s)/objectives To estimate clinical cure and TV eradication rates in a large cohort of $T$. vaginalis cases.

Methods All positive T. vaginalis NAAT results (TV TMA, Hologic) were identified between January 2013 and September 2015. Data were collected from our electronic patient record system, including clinical features, treatment regimen and TOC results, if performed.

Results 557 cases were identified in 500 patients $(78.2 \%$ female; 82.2\% Black African/Caribbean/mixed ethnicity; 8.8\% HIV+). Infection was symptomatic in $47.3 \%$ (53.7\% females, $24.5 \%$ males). Baseline wet mount microscopy was positive in $65.6 \%$. TOC was performed in $72.4 \%$ (median time to TOC 4.1 weeks, IQR 2.3-7.6 weeks). 77.2\% demonstrated parasitological clearance following a single treatment course. Cure rates were $70-80$ for all regimens, significantly higher in males $(85.5 \%$ vs $66.9 \%$, $\mathrm{p}<0.01)$.

Discussion/conclusion We see a significant asymptomatic, microscopy-negative burden of $T$. vaginalis infection. Lower clearance rates in women suggest azole-resistant strains may be prevalent. Based on NAAT results, cure rates are lower than expected, and relatively constant TMA positivity rate beyond 2 weeks suggests treatment failure is responsible rather than re-infection or timing of TOC. Further UK studies on treatment efficacy and molecular epidemiology are warranted.

\section{O025 BEHAVIOURAL FACTORS ASSOCIATED WITH HPV VACCINE ACCEPTABILITY AMONGST MEN WHO HAVE SEX WITH MEN IN THE UNITED KINGDOM}

${ }^{1}$ Tom Nadarzynski ${ }^{*}$, ${ }^{1}$ Helen Smith, ${ }^{1,2}$ Daniel Richardson, ${ }^{1}$ Stephen Bremner, ${ }^{1}$ Carrie Llewellyn. ${ }^{1}$ Brighton and Sussex Medical School, Brighton, UK; ${ }^{2}$ Brighton and Sussex University NHS Trust, Brighton, UK

\subsection{6/sextrans-2016-052718.24}

Background Men who have sex with men (MSM) are selected for Human Papillomavirus (HPV) vaccination due to their higher risk of genital warts and anal cancer.

Aim To examine HPV vaccine acceptability amongst MSM in the UK.

Methods Using Facebook advertisements, MSM were recruited for an online survey measuring motivations for HPV vaccination. Logistic regression was performed to identify predictors of HPV vaccine acceptability at baseline, after receiving information about HPV vaccination, and four weeks later.

Results Out of 1508 MSM (median age $=22$, range: 15-63) 19\% knew about HPV. While only 55\% of MSM would be willing to ask for the HPV vaccine, $89 \%$ would accept it if offered by a healthcare professional (HCP). Access to sexual health clinics $[\mathrm{OR}=1.82,95 \%$ CI 1.29-2.89], the disclosure of sexual orientation to an HCP [OR $=2.02$, CI 1.39-3.14] and HIV-positive status [OR $=1.96$, CI 1.09-3.53] positively predicted HPV vaccine acceptability. After receiving the information, perceptions of HPV risk [OR = 1.31, CI 1.05-1.63], HPV infection severity $[\mathrm{OR}=1.89, \mathrm{CI} 1.16-3.01), \mathrm{HPV}$ vaccination benefits $[\mathrm{OR}=1.61, \mathrm{CI} 1.14-3.01], \mathrm{HPV}$ vaccine effectiveness [OR $=1.54$, CI 1.14-2.08], and the lack of perceived barriers to $\mathrm{HPV}$ vaccination $[\mathrm{OR}=4.46, \mathrm{CI} 2.95-6.73$ ] were also associated with acceptability.

Discussion Although nearly half of MSM would not actively pursue HPV vaccination, the vast majority would accept the vaccine if recommended by HCPs. MSM need to be informed about 